\title{
Application of computer simulation in the study of infectious disease transmission mechanism
}

\author{
Chengzhen Zhao ${ }^{1, *}$, Hui Zhao ${ }^{1}$, and Xun liang ${ }^{2}$ \\ ${ }^{1}$ School of Economics, Beijing Wuzi University, Beijing, 101149, China \\ ${ }^{2}$ School of Information, Renmin University of China, Beijing, 100872, China
}

\begin{abstract}
Major infectious diseases have exerted a serious influence on people's lives. Through quantifying the effect of prevention and control, we can deeply understand the transmission mechanism of infectious diseases. This paper estimates the intensity of detection, the degree of isolation and other indicators, and analyzes the influence mechanism of these indicators on the scale of the epidemic, using computer programming to simulate the extended dynamics model of infectious diseases, based on the infectious disease in Hubei. The mortality rate and recovery rate, according to the data of Hubei, in the model are set as time variables, and the threshold is set at the same time. As a result, the improved analysis mechanism of the model will get more realistic simulation prediction results. It is concluded that isolation measures can effectively control the scale of the epidemic, but there is a phenomenon of marginal utility degression with excessively strict isolation measures by analysing and comparing. The increasing detection efforts will reduce the epidemic duration of the later stage, accelerating the arrival of the epidemic peak, although the peak will be slightly larger. All in all, we can comprehensively consider the testing cost and maintain a moderate detection intensity.
\end{abstract}

\section{Introduction}

As a recent major epidemic disease, the COVID-19 epidemic poses a threat to people's health in many countries. The main outbreak area of China is Hubei Province, so we can summarize experience from the epidemic data of Hubei, and provide some reasonable suggestions for epidemic prevention and control in other parts of the world. Whether the measures are effective and how effective they are, they need to be evaluated by combining actual data and rigorous theoretical models. Therefore, this paper, denoting the detection intensity, isolation and other measures as numerical indicators, and using the actual epidemic data to estimate the values of these indicators, constructs the mathematical model of infectious diseases. In this way, various measures in different regions can be compared intuitively, we can also observe the relationship between the implementation of various measures and the effect of prevention and control, and predict the trend of epidemic in the future, to provide references for the formulation of relevant decisions.

\footnotetext{
* Corresponding author: zhaochengzhenabc@163.com
} 


\section{Related work}

In 1760 , Bernoulli $\mathrm{D}$ established a model to study the effect of vaccination on smallpox transmission [1]. Hamer W $\mathrm{H}$ established a discrete-time model to understand the recurrence of measles epidemic and creatively assumed the incidence, that is, the number of new cases per unit of time depends on the product of the number of susceptible and diagnosed cases [2]. Ross $\mathrm{R}$ established a differential equation model of malaria as a host disease and studied the transmission behavior of malaria between mosquitoes and people [3]. Subsequently, other deterministic infectious disease models have also been proposed. At the beginning of the 20th century, with the gradual rise of mathematical epidemiology, the study of infectious disease models is deeper and deeper. Kermack W O et al. divided the population in the study area into three categories by studying the Black death and the plague, namely, Susceptible, Infective, and Recovered [4]. Then, he put forward the famous SIR warehouse model, which is suitable for describing infectious diseases transmitted by viruses. Subsequently, Kermack W O established the SIS warehouse model based on the SIR warehouse model, and put forward the famous threshold theorem, but many infectious diseases usually have a certain incubation period before onset, such as the incubation period of AIDS is generally 2 to 12 years, the incubation period of rabies is generally 1-3 months [5]. Thus, considering the factor of incubation period, researchers adding a new group of infectious diseases, Exposed, put forward the SEIR model based on the SIR model. SEIR model has been applied and studied in many fields in our country. Zhou Lingli et al. comprehensively considered the influence of age structure on the epidemic process of infectious diseases with exposed period, and analyzed the stability of the equilibrium point based on the SEIR discrete model [6]. Wang Bingjie compared the effectiveness of vaccination and treatment, based on SEIR infectious disease model, showing that vaccination can control infectious diseases more effective than treatment in both exposed period and infection period, and the mixing strategies are more effective when they are used at the same time [7]. Andrew S Azman and Francisco J Luquero summarized and evaluated China's prevention and control experience, arguing that positive interventions across China, including city closures, active surveillance of cases, rapid investment to improve testing capacity, isolation of cases and high-risk groups, treatment of severe cases, and mandatory wearing of masks, may lead to a significant reduction in transmission [8]. These measures provide a reference for other countries to design responses to COVID-19 according to their experience. The research results of Zhang Juanjuan et al. showed that strict control measures, movement restrictions and the improvement of population awareness may be part of the reasons for interruption of SARS-CoV-2 transmission outside Hubei Province [9].

\section{Construction of infectious disease model}

This paper will take the SEIR model as the benchmark, and make improvements on this basis. According to the study of this paper, the population within the epidemic range of infectious diseases is divided into five types.

The susceptible: marked as St, means people who are not infected but may be infected now. The exposed: people who are marked as Et, and indicates that they have been infected at time $t$, but have not been diagnosed by testing and can infect others. The infectious: people who are marked as It, and indicates that they have been infected with the virus at time $t$, have been tested and diagnosed, and have been centrally treated, and can infect others at the same time. The recovered: marked as Rt, indicates that people who recover at time $t$ and will not be infected again will no longer affect the dynamic behavior of the system. The dead: marked as Dt, it means that the people who die of infectious diseases at time $t$ will no longer affect the dynamic behavior of the system. 
Assuming that the total population of the epidemic area is N, which remains unchanged, regardless of the migration and emigration of the population, ignoring the impact of natural birth and deaths caused by non-infectious diseases on $\mathrm{N}$, that is:

$$
N=S_{t}+E_{t}+I_{t}+R_{t}+D_{t}
$$

It is assumed that the number of susceptible people transferred to exposed persons per unit time is positively correlated with the number of the susceptible people, the exposed people, and the infectious people. The number of transfers per unit time is:

$$
\beta_{1}\left(\alpha_{1} S\right)\left(\alpha_{2} I\right) / N+\beta_{2}\left(\alpha_{1} S\right)\left(\alpha_{1} E\right) / N
$$

Among them, $\beta_{1}$ represents the nonlinear infection coefficient of the infectious people, $\beta_{2}$ represents the nonlinear infection coefficient of the exposed people, and $\alpha_{1}$ represents the exposure degree of the susceptible and the exposed people, which is determined by the restriction mobile contact degree of the susceptible and exposed population, and $\alpha_{2}$ represents the exposure degree of the infectious people, which is determined by the degree of isolation treatment of the infectious people when centralized treatment. The lower the values of $\alpha 1$ and $\alpha_{2}$ are, the stricter the isolation measures.

\subsection{Model propagation mechanism}

In the transmission mechanism, $\sigma$ represents the proportion of exposed persons transformed into infectious people per unit time, based on the reciprocal of the average incubation period of infectious diseases, and the specific size is related to the intensity of medical detection in different areas. To a certain extent, the greater the detection intensity is, the bigger the $\sigma$ becomes. $\Upsilon$ represents the one-day recovery rate of infectious people in unit time, hereinafter referred to as the recovery rate or cure rate. $v$ represents the one-day mortality rate of infectious people per unit time, hereinafter referred to as the mortality rate. The differential dynamic equations of the SEIR model constructed in this paper are as follows:

$$
\left\{\begin{array}{l}
d S / d t=-\beta_{1} \alpha_{1} \alpha_{2} S I / N-\beta_{2} \alpha_{1}^{2} S E / N \\
d E / d t=\beta_{1} \alpha_{1} \alpha_{2} S I / N+\beta_{2} \alpha_{1}^{2} S E / N-\sigma E \\
d I / d t=\sigma E-\gamma I-v I \\
d R / d t=\gamma I \\
d D / d t=v I \\
N=S+E+I+R+D
\end{array}\right.
$$

\subsection{Parameter setting}

The mortality rate and recovery rate are set as variables in the model, in order to be more in line with the reality. Theoretically, since one patient can infect 2-3 people, it is recorded as $\mathrm{Cn}$, and $\mathrm{Cn}$ is taken as 2 . If the incubation period is distributed averagely from 0 to 14 days, the average incubation period is 7, and the nonlinear infection coefficient $\beta 2$ of the exposed people is estimated to be the value of $\mathrm{Cn} / 7$, that is, 0.286 . Because the infectious people will be concentrated, the chance of close contact with other people is much less than that of the exposed people. In this paper, it is assumed that the chance of contact between the infectious people and other people is 0.02 times than that of the exposed people. The estimated value of the nonlinear transmission coefficient $\beta 1$ of the infectious people is 
0.006. The base value of $\sigma$ is set as the reciprocal of the average incubation period, that is, 0.143 .

\section{The influence of various factors on the predicted value of epidemic situation}

This part mainly analyzes the influence mechanism of these factors on the simulation results: the ratio of exposed persons transformed into infectious persons ( $\sigma$ ) per unit time, the exposure degree of susceptible and exposed persons $\left(a_{1}\right)$, the exposure degree of infectious persons $\left(\alpha_{2}\right)$, and the initial number of exposed persons $\left(E_{0}\right)$. $\sigma$ can reflect the intensity of medical detection of people in epidemic areas of infectious diseases, $a_{1}$ can reflect the effect of isolation measures for susceptible and exposed populations, $a_{2}$ can reflect the effect of isolation measures for centralized treatment of patients. E0 reflects the current number of exposed persons scattered in the epidemic area.

February 6, 2020 is selected as the starting date for the analysis of the epidemic situation in Hubei Province, and the initial values of several types of population are the actual data on the starting date of the analysis. The resident population of Hubei Province in 2019 is 59.27 million. Let $\mathrm{N}$ be $59,270,000$. The initial value of $\mathrm{I}\left(\mathrm{I}_{0}\right)$ is the number of diagnosed cases, 20,677. The initial value of $\mathrm{R}\left(\mathrm{R}_{0}\right)$ is the cumulative number of rehabilitations, 817 . The initial value of $\mathrm{D}\left(\mathrm{D}_{0}\right)$ is the cumulative number of deaths, 618 . And the initial value of $E\left(E_{0}\right)$ can' $t$ be obtained directly. We can estimate the value of $E_{0}$ backwards according to the influence mechanism of $\mathrm{E}_{0}$ on the model and the known relevant data. The initial value of $\mathrm{S}: \mathrm{S}_{0}=\mathrm{N}-\mathrm{E}_{0}-\mathrm{I}_{0}-\mathrm{R}_{0}-\mathrm{D}_{0}$. In order to facilitate the observation of the influence mechanism of various factors, we use the control variable method, that is, when analyzing one of the factors, the other factors take the default value. Let the default value of $\sigma$ be 0.143 , the default value of $\alpha_{1}$ and $\alpha_{2}$ be 0.5 and the default value of $E_{0}$ be 50,000 .

\subsection{The influence of $\sigma$ on the epidemic situation}

Using MATLAB to simulate the model, we can get the predictive values of the number of diagnosed cases and cumulative deaths under different $\sigma$ values, as shown in Figures 1 and 2.

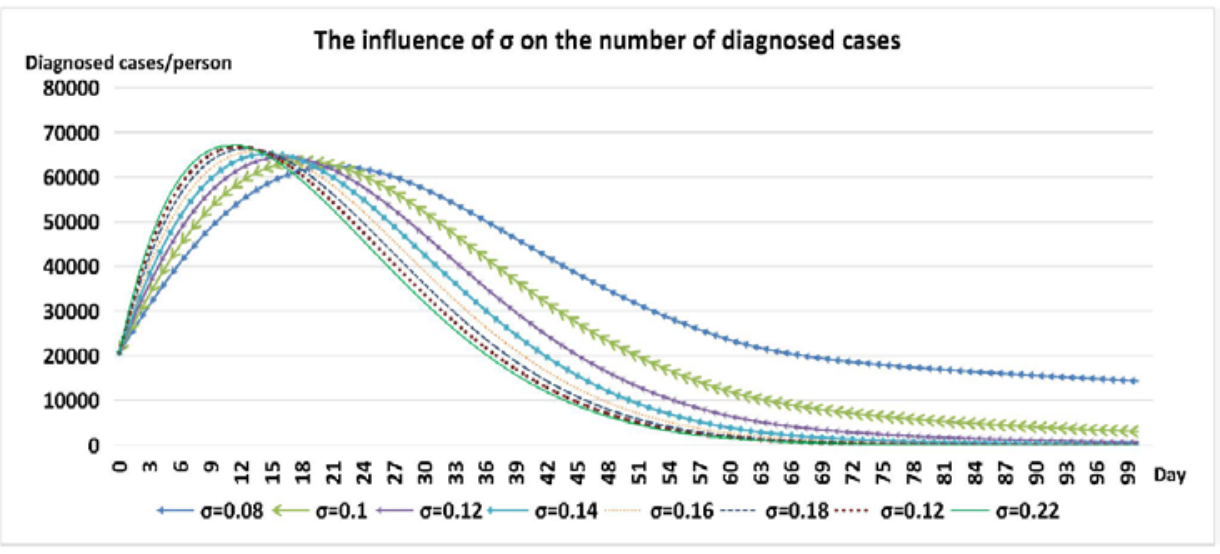

Fig. 1. The impact of the proportion of exposed persons transformed into infectious persons per unit of time on the number of remaining infectious cases. 
The larger the value of $\sigma$ becomes, the greater the intensity of medical detection of infectious diseases is. It can be found from Figure 1 that as $\sigma$ increases from 0.08 to 0.22 , the number of diagnosed cases will peak earlier and decrease earlier, which means that increased testing can shorten the duration of the epidemic. We also draw two more important conclusions: for one thing, it is that the increase in testing has not played a positive role in reducing the peak value, the scale of the epidemic, of remaining infectious cases, and making the peak slightly larger. But the arrival time of the peak will be advanced; for another, with the increase of $\sigma$, its marginal impact on the number of infectious cases decreases. Therefore, considering the cost and effectiveness of testing measures, the work on improving the intensity of testing can be maintained at a moderate level in various regions.

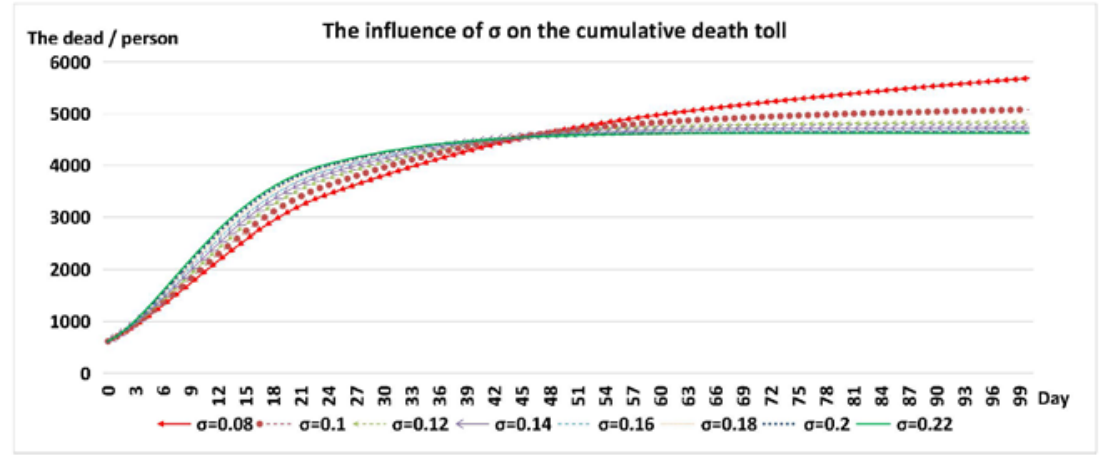

Fig. 2. The impact of the proportion of exposed persons converted into infectious persons per unit of time on the cumulative number of deaths.

It can be found from Figure 2 that with the increase of $\sigma$ from 0.08 to 0.22 , the cumulative death toll will reach the convergence value earlier, and the final death toll can be reduced at the same time. It means that increasing testing may be reflected in the increase of the deaths in the early stage, but the final total deaths will be reduced. Similar to the impact of remaining infectious cases, the marginal impact on the cumulative number of deaths decreases with the increase of $\sigma$. It also shows that in the situation of limited resources, the appropriate improvement of detection intensity can achieve better results.

\subsection{Effect of $\alpha_{1}$ on epidemic situation}

The predicted values of remaining infectious cases and cumulative deaths under different $a_{1}$ values are shown in Figures 3 and 4.

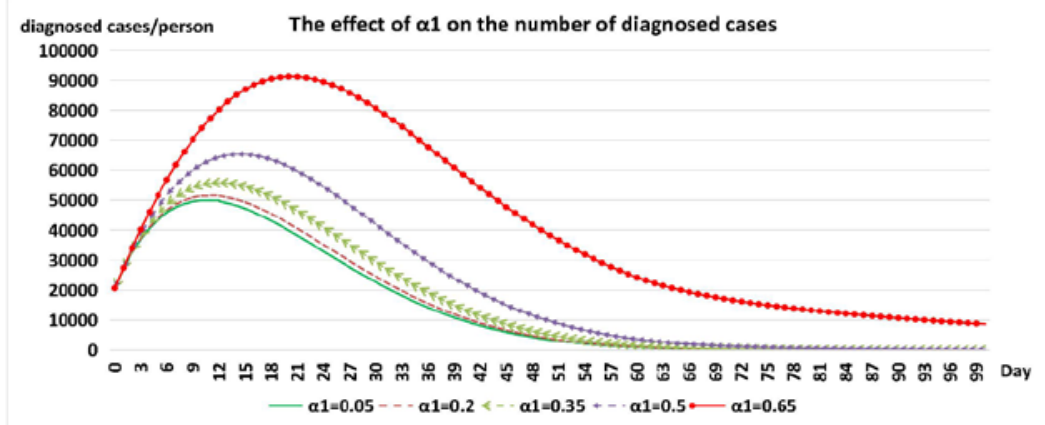

Fig. 3. The impact of exposure of susceptible and exposed persons on the number of remaining infectious cases. 
The smaller the value of $\alpha_{1}$ is, that is, tighter isolation measures, the less the exposure of the susceptible and exposed persons will be. With the stricter quarantine measures, the peak value will decrease significantly, and the size of infectious cases will be controlled better throughout the epidemic period. Therefore, the positive impact of isolation measures for susceptible and exposed persons is greater than that of strengthening testing. But at the same time, the marginal influence of isolation measures is decreasing. The size of the remaining infectious cases number has been reduced caused by the decrease of $\alpha_{1}$ from 0.65 to 0.5 , which is larger than that of influencing by the decrease of $\alpha_{1}$ from 0.5 to 0.05 . Thus, for areas where it is difficult to bear the economic losses caused by complete home isolation, appropriate isolation measures can be taken, such as maintaining a certain social distance, wearing masks and so on, which can play a greatly positive role in the control of epidemic.

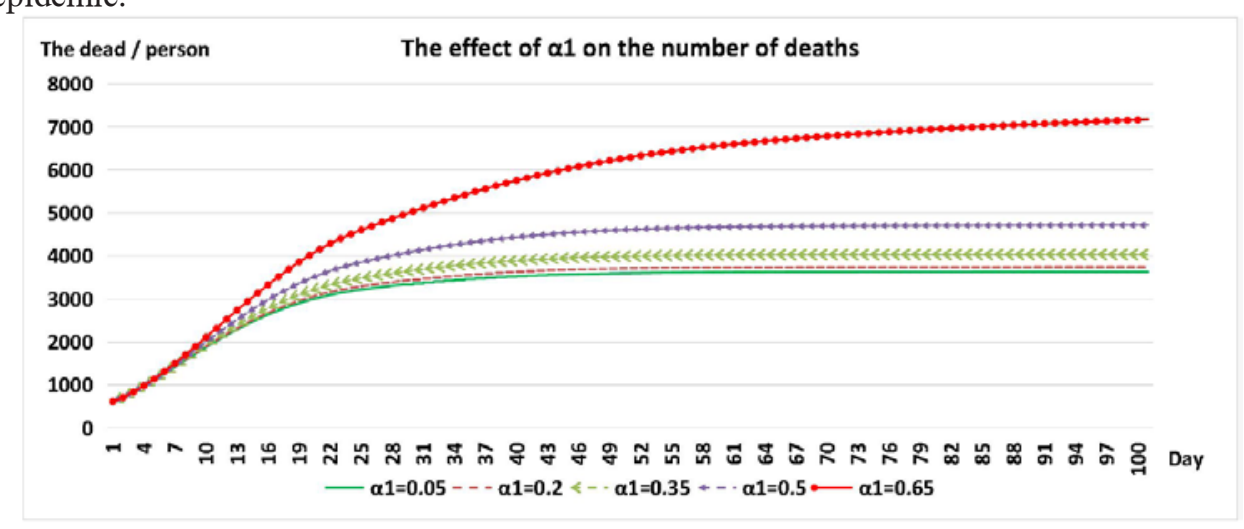

Fig. 4. The impact of exposure of susceptible and exposed persons on the number of deaths.

As can be seen from Figure 4, unlike the influence of $\sigma$ on the deaths, the decrease of $\alpha_{1}$ will significantly reduce the death toll throughout the epidemic period, not only in the final death toll, but also by a greater extent than $\sigma$. Considering that the marginal influence of $\alpha_{1}$ is decreasing obviously, the death toll can be greatly reduced by taking appropriate isolation measures in the relevant areas.

\section{Conclusions}

\subsection{The intensity of isolation measures should be properly strengthened.}

It can be found that the smaller the exposure of susceptible and exposed people controlled by $a_{1}$, the better the control scales of remaining infectious cases are. However, from the influence mechanism of isolation measures, it can also be found that due to the phenomenon of diminishing marginal utility of isolation measures, the isolation measures in Hubei Province are too strict, resulting in small marginal benefits. Isolation measures need to sacrifice social and economic development, so the implementation of isolation measures in various regions need to be appropriately strengthened, but too strict measures is not necessary, and an appropriate level can achieve better result.

\subsection{The intensity of testing should be kept at a moderate level.}

In this paper, when studying the influence mechanism of the detection intensity coefficient of epidemic situation, it is found that with the strengthening detection intensity, the peak 
value of the number of infectious cases will come ahead of schedule, and the duration of the whole epidemic situation will be shortened correspondingly, but the detection intensity also has the phenomenon of diminishing marginal utility. Therefore, in the prevention and control of the epidemic, we can appropriately increase the intensity of detection in order to control the development of the epidemic faster. Considering the high cost of testing, each region can synthesize its own situation and maintain a moderate intensity of testing.

This work was financially supported by The Ministry of education of Humanities and Social Science project of China (No.19YJC790189), and Education Commission Social Science Fund of Beijing, China (No.SM202010037006).

\section{References}

1. BERNOULLI D.Essai d'une nouvelle analyse de la mortalité causée par la petite vérole et des avantages de l'inoculation pour la prévenir[D].Paris:Académie Royale des Sciences, 1760:1-45.

2. HAMER W H.The milroy lectures on epidemic diseases in England - - the evidence of variability and of persistency of type[J].The Lancet, 190,167(4305):569-574.

3. ROSS R.The mathematics of malaria[J].British medical journal,1911,1(2626):1023.

4. KERMACK W O, MCKENDRICK A G.Contribution to the mathematical theory of epidemics[C].Proceedings of the Royal Society of London.Series A,1927,115:700-721.

5. KERMACK W O, MCKENDRICK A G.Contribution to the mathematical theory of epidemics.II.the problem of endemicity[C].Proceedings of the Royal Society of London,Series A, 1932,138:55-83.

6. Zhou Ling-li,Sun Guang-hui,LI Ai-qin.Global Analysis of Discrete SEIR Model with exposed Period[J].Mathematics in practice and theory,2010,40(07):136-143.

7. Wang Bing-jie.Control strategies of an SEIR epidemic model with infectious force in exposed period[J].Journal of Northeast Normal University(Natural Science Edition),2014,46(01):28-32.

8. Andrew S Azman,Francisco J Luquero.From China:hope and lessons for COVID-19 control.Lancet Infect Dis 2020;published Online April 2.https://doi.org/10.1016/S1473-3099(20)30264-4.

9. Juanjuan Zhang,Maria Litvinova,Wei Wang,et al.Evolving epidemiology and transmission dynamics of coronavirus disease 2019 outside Hubei province,China:a descriptive and modelling study.Lancet Infect Dis 2020;published Online April 2.https://doi.org/10.1016/S1473-3099(20)30230-9. 